\title{
Spatial selectivity in vision: Field size depends upon noise size
}

\author{
PHILIP M. MERIKLE and NANCY J. GOREWICH \\ University of Waterloo, Waterloo, Ontario N2L 3G1, Canada
}

\begin{abstract}
The size of the visual attentional field was estimated by a modified Stroop task that required the naming of centrally fixated color patches. Noise material consisted of incompatible color words located above and below the color patches, and the target-noise separation was either .5 or $2.5 \mathrm{deg}$ of visual angle. In addition, the size of the noise material subtended either .24 (small) or $.57 \mathrm{deg}$ (large) of vertical visual angle. Relative to comparable neutral noise conditions, the large noise material slowed color-naming reaction times (RTs) at both target-noise separations, but the small noise material only affected RT when the tareget-noise separation was $.5 \mathrm{deg}$ of visual angle. Assuming that the effects of noise indicate the extent of the attentional field, these results suggest that any estimate of attentional field size is critically dependent upon the size of the noise material.
\end{abstract}

It is now well established that attentional selectivity to a specific spatial location is very imperfect. In an extensive series of studies, Eriksen and his associates (Colegate, Hoffman, \& Eriksen, 1973; Eriksen \& Eriksen, 1974; Eriksen \& Hoffman, 1972, 1973) have demonstrated that the reaction time (RT) to respond to a designated target letter is affected by the character of the adjacent material (noise) presented in the visual field. These effects of noise are easily demonstrated under conditions that involve cuing of the location of the attended letter prior to display onset. Even when a target letter is presented in a known centrally located position on every trial, the RT to respond is influenced by the flanking noise letters (Eriksen \& Eriksen, 1974). Overall, the results of the studies reported by Eriksen and his associates provide unequivocal evidence that it is impossible to tune visual attention so that only the information presented in a precise spatial location receives perceptual analysis.

Two different explanations have been proposed to account for the imprecise nature of spatial selectivity. The explanation favored by Eriksen (e.g., Eriksen \& Eriksen, 1974) is that selection of information from the visual field is limited by the degree to which attention can be focused. This spatial focusing view of attention is based upon the results of studies in which the separation between target letters and the noise material has been systematically varied (e.g., Eriksen \& Eriksen, 1974; Eriksen \& Hoffman, 1972, 1973). A consistent finding in these studies is that there is little, if any, effect of the noise material when the spacing is $1 \mathrm{deg}$ of visual

This research was supported by Grant APA-231 from the National Research Council of Canada to the first author. The participation of the second author was made possible by an R. H. Walters' assistantship. Requests for reprints should be sent to Philip M. Merikle, Department of Psychology, University of Waterloo, Waterloo, Ontario N2L 3G1, Canada. angle or greater. These results have led Eriksen (e.g., Eriksen \& Eriksen, 1974) to propose that attention may be focused on an area of visual field with a radius of approximately $1 \mathrm{deg}$ of visual angle. According to this proposal, all information falling within $1 \mathrm{deg}$ of visual angle from the point of fixation receives at least some perceptual analysis, while information outside this focus can be successfully ignored.

A considerably different explanation for the imprecise nature of spatial selectivity has been offered by Gatti and Egeth (1978). Consistent with recent proposals emphasizing the lack of attentional control over initial perceptual analysis (e.g., Shiffrin, McKay, \& Shaffer, 1976), they have suggested that all material in the visual field is processed, if possible. According to their view, the major factor limiting perceptual analysis of noise material is visual acuity. Therefore, the decreased effect of noise observed by Eriksen and his associates when the target-noise separation was increased can be attributed simply to a correlated decrease in the quality of the stimulus information for the noise material.

Gatti and Egeth (1978) have supported their proposed explanation by the results of an experiment in which they estimated the size of the visual attentional field through the use of a modified Stroop (1935) colorword task. The target on each trial was a centrally fixated color patch, and the noise material was an incompatible color name printed above and below the location of the color patch. In order to compensate for the reduction in acuity with increased target-noise separation, Gatti and Egeth used considerably larger letters for the noise material than have ever been employed by Eriksen and his associates. The results showed that the RT to name the color patches was increased even when the incompatible color words were separated from the color patches by as much as $5 \mathrm{deg}$ 
of visual angle, which was the maximum target-noise separation used in the experiment. These results suggest that estimates of the size of the visual attentional field may be critically dependent upon the size of the noise material. If this conclusion is accepted, then the view proposed by Eriksen and his associates that attention can be focused exclusively on a very limited area of the visual field must be rejected.

One problem encountered when making comparisons between the Gatti and Egeth (1978) study and the experiments reported by Eriksen and his associates is that the different investigators used both different tasks and different-sized noise materials. Either of these factors may have contributed to the different estimates for the size of the visual attentional field. What is needed in order to decide whether attention can be focused exclusively on a limited area of the visual field is a single study that involves systematic variation of both the size of the noise material and the target-noise separation. Such was the purpose of the present study.

The task used in the present experiment was the modified Stroop task employed by Gatti and Egeth (1978), and the incompatible color words were located at two different distances from the centrally fixated color patches. In order to test whether the estimate of attentional field size is dependent upon the size, and therefore the resolution, of the noise material, the color words were printed in two different-sized letters. If the spatial focusing view proposed by Eriksen and his associates is correct, then the physical size of the noise material should not influence the obtained estimates of attentional field size. On the other hand, if the major limitation on the processing of noise material is the visual system's ability to resolve the stimulus information, as suggested by Gatti and Egeth, then the estimates of attentional field size should be dependent upon the size of the noise material.

\section{METHOD}

\section{Materials and Design}

Two sets of 24 stimulus displays were constructed. All displays contained a centrally located, $.5-\mathrm{cm}^{2}$ color patch on a white card, and in each set, there were eight displays containing each of the following colors: red, blue, and green. For the eight displays in each set containing a specific color, there were two displays representing each of the following conditions: (1) incompatible-narrow, (2) neutral-narrow, (3) incompatiblewide, and (4) neutral-wide. In the incompatible conditions, a color word that named a color different from the color patch was located above and below the location of the color patch. The neutral conditions had lowercase ls (e.g., 1111) in positions comparable to those occupied by the letters in the color words. For the narrow target-noise separation conditions, the center-tocenter distance between a color patch and either the color words or the neutral material was $1.1 \mathrm{~cm}$; for the wide target-noise separation conditions, the comparable distance was $5.3 \mathrm{~cm}$. When the displays were viewed through the tachistoscope, these distances were equivalent to .5 and $2.5 \mathrm{deg}$ of visual angle, respectively.

The two sets of displays were distinguished by the size of the material presented above and below the color patches.
For one set of displays (small noise condition), the letters had a vertical dimension of $.5 \mathrm{~cm}$ (Letraset 287); for the second set of displays (large noise condition), the vertical dimension of the letters was $1.2 \mathrm{~cm}$ (Letraset 760). The letters for both sets of displays had the same typeface and differed only in terms of overall size. When viewed through the tachistoscope, the small and large letters subtended approximately .24 and $.57 \mathrm{deg}$ of vertical visual angle, respectively.

\section{Procedure}

All materials were presented by means of a Scientific Prototype three-field tachistoscope (Model GB). The luminance of all fields, as measured with a blank white card in each field by a Pritchard photometer (Model 1970-PR), was approximately $60 \mathrm{~cd} / \mathrm{m}^{2}$.

Each subject was instructed to fixate a small dot located in the center of the visual field. The position of the fixation point corresponded to the centers of the color patches. The subjects were told to identify each color as quickly as possible and to ignore the material printed above and below each color patch. Prior to the beginning of the experiment proper, each subject was shown three practice displays containing each of the color patches. The practice trials insured that the subjects could identify each of the colors and allowed the experimenter to adjust the sensitivity of the voice key used to determine vocal RT for color naming.

Following completion of the practice trials, there were 144 experimental trials divided into three blocks of 48 trials. Each of the 24 displays in the two sets was presented once in each trial block. The presentation order was determined by shuffling the deck of cards containing the displays prior to the beginning of each trial block. Each display was presented for $200 \mathrm{msec}$, and RT was measured from display onset. The presentation of each display was preceded by a verbal ready signal from the experimenter.

\section{Subjects}

The eight subjects (three males) were undergraduates at the University of Waterloo who volunteered to participate.

\section{RESULTS AND DISCUSSION}

For each subject, the median RTs across the three presentations of each display were determined. The means of the medians for the six displays representing each experimental condition were then determined, and the resultant scores were subjected to a 2 by 2 by 2 analysis of variance to evaluate the effects of noise material (incompatible or neutral), target-noise separation (narrow or wide), and noise size (small or large).

Figure 1 shows the mean RT for each of the experimental conditions. The critical data involve comparisons between the incompatible and neutral noise conditions for each size noise material at each target-noise separation. The logic behind the experiment dictates that evidence for the analysis of noise material is obtained when the mean color-naming RT is slower under an incompatible noise condition than under a comparable neutral noise condition. As indicated by an inspection of the figure, evidence for the analysis of noise material was obtained for three out of the four possible comparisons. When the size of the noise material was small, the data indicate that the noise material was only analyzed when there was a narrow separation $(.5 \mathrm{deg})$ between the target and the noise material. However, for the large- 


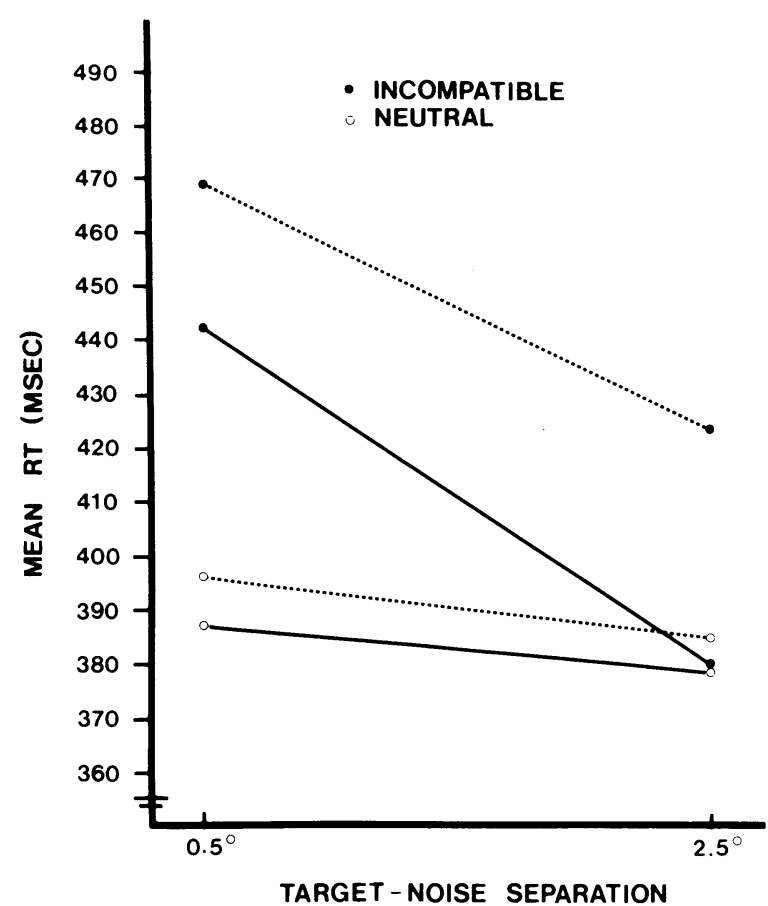

Figure 1. Mean color-naming RT for large (broken line) and small (solid line) noise material.

sized noise material, the data indicate that the noise material was analyzed under both the narrow $(.5 \mathrm{deg})$ and the wide $(2.5 \mathrm{deg})$ target-noise separations.

The above pattern of results led to the following outcomes from the analysis of variance. Overall, the analysis indicated that color-naming RTs were (1) slower for incompatible than for neutral noise material $[F(1,7)=8.28, p<.025]$, (2) slower for narrow than for wide target-noise separations $[F(1,7)=17.23$, $\mathrm{p}<.01]$, and (3) slower for large- than for small-sized material $[F(1,7)=12.35, p<.01]$. The magnitude of the difference between the incompatible and the neutral noise material was also found to vary significantly as a function of both the size of the noise material $[F(1,7)=5.93, p<.05]$ and the target-noise separation $[F(1,7)=10.79, p<.025]$. In addition, the threefactor interaction was a significant source of variance $[F(1,7)=5.65, p<.05]$, indicating that the incompatibleneutral noise difference varied as a joint function of noise size and target-noise separation.

The nature of the three-factor interaction becomes clear when the data for the narrow and the wide targetnoise separations are considered individually. As indicated in Figure 1 for the narrow target-noise separation, there was a large incompatible-neutral noise difference with both the large and the small noise material. On the other hand, for the wide target-noise separation, an incompatible-neutral noise difference only occurred with the large noise material. Thus the data indicate that both the large and the small noise material were analyzed under the narrow spacing conditions, but that only the large noise material was analyzed under the wide spacing condition. These differential effects of the large and the small noise material under the two spacing conditions were confirmed by separate analyses of variance on the scores for the narrow and the wide spacing conditions. For the narrow target-noise separation, the analysis gave no indication that the magnitude of the incompatible-neutral noise difference varied as a function of noise size $[F(1,7)<1]$. However, for the wide target-noise separation, the analysis revealed a highly significant interaction between noise material and noise size $[F(1,7)=20.42, p<.01]$.

The present data are consistent with the previous results reported both by Eriksen and his associates (e.g., Eriksen \& Eriksen, 1974) and by Gatti and Egeth (1978). Eriksen and his associates have always used noise material that is comparable in size to the small noise material used in the present experiment. If only the data for the small noise conditions in the present experiment are considered, then it is possible to conclude, as Eriksen and his associates have done, that attention can be focused on an area of the visual field as small as $1 \mathrm{deg}$ of visual angle in radius. This conclusion would follow from the finding that the small noise material only affected RT when the target-noise separation was $.5 \mathrm{deg}$ of visual angle. However, the data for the large noise conditions, which are consistent with the findings reported by Gatti and Egeth (1978), indicate that this conclusion is incorrect. The data show that the large noise material was analyzed even when the target-noise separation was $2.5 \mathrm{deg}$ of visual angle. If attention could be focused exclusively on a particular spatial location so that information outside this focal area was excluded from analysis, then in the present experiment, noise material size should not have influenced the estimate of the size of the visual attentional field.

The straightforward conclusion suggested by the present data is that any estimate of the size of the visual attentional field is critically dependent upon the size of the noise material. In addition, it is reasonable to assume that the major limitation on the processing of noise material is visual acuity, as suggested by Gatti and Egeth (1978), and not the ability to focus attention upon a small area of the visual field, as suggested by Eriksen and his associates. On the other hand, while the present data are also consistent with recent proposals emphasizing the absence of attentional control over initial perceptual analysis (e.g., Shiffrin et al., 1976), they do not have a direct bearing upon the question of whether attention facilitates initial perceptual processing. As noted by Egeth (1977), it is certainly possible that the initial processing of the noise material was attenuated in the present task by the demand to focus attention upon a specific spatial location. The only conclusions that can be drawn from the present data are that the visual attentional field is much larger than claimed by Eriksen and his associates, and that any estimate of the size of the visual attentional field is dependent upon the size of the noise material. 


\section{REFERENCES}

Colegatr,, R. L., Hoffman, J. E., \& Eriksen, C. W. Selective encoding from multielement visual displays. Perception \& Psychophysics, 1973, 14, 217-224.

EgETH, H. E. Attention and preattention. In G. H. Bower (Ed.), The psychology of learning and motivation (Vol. 11). New York: Academic Press, 1977.

ERIKSEN, B. A., \& Eriksen, C. W. Effects of noise letters upon the identification of a target letter in a nonsearch task. Perception \& Psychophysics, 1974, 16, 143-149.

Eriksen, C. W., \& Hoffman, J. E. Temporal and spatial characteristics of selective encoding from visual displays. Perception \& Psychophysics, 1972, 12, 201-204.
Eriksen, C. W., \& Hoffman, J. E. The extent of processing of noise elements during selective encoding from visual displays. Perception \& Psychophysics, 1973, 14, 155-160.

Gatti, S. V., \& Egeth, H. E. Failure of spatial selectivity in vision. Bulletin of the Psychonomic Society, 1978, 11, 181-184.

Shiffrin, R. M., McKay, D. P., \& Shaffer, W. O. Attending to forty-nine spatial positions at once. Journal of Experimental Psychology: Human Perception and Performance, 1976, 2, 14-22. Stroop, J. R. Studies of interference in serial verbal reactions. Journal of Experimental Psychology, 1935, 18, 643-662.

(Received for publication August 1, 1979.) 